\title{
Towards a robotic personal trainer for the elderly
}

\author{
J. A. Rincon ${ }^{1}$, A. Costa ${ }^{2}$, P. Novais ${ }^{2}$, V. Julian ${ }^{1}$, C. Carrascosa ${ }^{1}$ \\ 1 Universitat Politècnica de València. Institut Valencià d'Investigació en \\ Intel-ligència Artificial (VRAIN) \\ \{jrincon@dsic.upv.es, vjulian@upv.es, carrasco@dsic.upv.es\} \\ 2 ALGORITMI Centre, Universidade do Minho, Portugal \\ \{acosta@di.uminho.pt,pjon@di.uminho.pt\}
}

Keywords: Assistant Robot, Emotion detection, Elderly

\begin{abstract}
The use of robots in the environment of the elderly has grown significantly in recent years. The idea is to try to increase the comfort and well-being of older people through the employment of some kind of automated processes that simplify daily work. In this paper we present a prototype of a personal robotic trainer which, together with a noninvasive sensor, allows caregivers to monitor certain physical activities in order to improve their performance. In addition, the proposed system also takes into account how the person feels during the performance of the physical exercises and thus, determine more precisely if the exercise is appropriate or not for a specific person.
\end{abstract}

\section{Introduction}

The substantial growth in the average age of the world's population leads to a larger number of older people than the rest of the age groups [1]. One of the major problems afflicting this population is its high level of sedentary life [2]. According to this, the development of cognitive assistants at home, and especially oriented to health and well-being of older people, is a very interesting field of study because it not only allows a daily monitoring of the health in a comfortable and fast way but also greatly lightens the workload that usually have hospitals and professionals [3]. These assistants usually provide telemedicine service, video calls, allowing the patient to be in contact with his doctor [4]. The advantage of this type of tasks performed on a daily basis at home is that they would allow a constant and exhaustive monitoring. So that by using models based on artificial intelligence techniques, with the large amount of available data, it could be possible to detect and anticipate possible problems or to suggest improvements in lifestyle habits.

Other characteristic that cognitive assistants currently may possess is the classification the emotional state of the human with whom they interact. As well as the ability to express an emotional state of their own, depending on the information they obtain from the surrounding environment. This, along with 
the rest, allows the users to feel a certain affinity with cognitive assistants. The information obtained by the assistant from this kind of interactions in combination with the large amount of data available, allow the assistants to classify and anticipate possible problems, and also to make personalized suggestions, in order to improve the well-being of the users.

This paper presents a cognitive assistant, specifically an assistant robot, that actuates as a personal trainer capable of recommending, detecting and classifying the physical activities [5], [6] performed by an elderly person. The robot is capable of interacting with humans, using voice and emotion detection as communication elements, recommending personalised physical activities. Moreover, while the elderly person is performing the exercises using a non-invasive sensor, the robot monitors whether the exercises are being done in an appropriate manner.

The rest of the paper is structured as follows: section 2 presents a related work section; section 3 explains the proposed approach; finally, section 4 gives some conclusions and possible future works.

\section{Related Work}

EmIR is inserted in mainly three domains: assistive robotics, and human activity recognition. There are several novel advances in these domains, either separately or in combination, contributing to the advancement of the state of the art. In this section we present some of these projects, serving as a comparative to EmIR features.

Assistive Robots Martinez-Martin et al. [7] provides a clear idea and several examples of assistive robotics and their features. Apart from them, we highlight three other projects. Caic et al. [8] presents the Vizzi robot. This robot has a very friendly aspect and is designed to interact with elderly people, proposing Exergames. The robot is able to navigate in flat surfaces and has an "emotion" displaying head. It also has speakers and microphones to capture and transmit voice commands. Currently it is not able to autonomously interact with the users or to interact with them, having an visual interface for a human caregiver to interact. In the same line is the work of [9], having the same physical features, it is more advanced on the interaction level and the unassisted movements. The robot is able to receive voice commands and perform autonomously certain tasks (that were pre-programmed) and navigate around a house, recording the paths and optimising them for future use. Lastly there is the SocialRobot [10], yet an advancement over the [9], being the most notable feature the ability to recognise human emotions and faces, and produce an empathetic interaction. It is also able to navigate in an home environment and visually identify users and objects.

Human Activity Recognition Martinez-Martin et al. [11-13] presents an rehabilitation system using a humanoid robot to be used at home. They use the robot's cameras to visually identify the user's physical movements, using deep learning 
methods. The robot is able to navigate around the house and identify the user. The information is made available caregivers so they can adjust the exercises accordingly. Using wearables, the work of Kwapisz [14] shows how to identify activities using just a mobile phone. This environment is perfect for a real application due to the low effort that users have in "wearing" these devices. While this specific work did not present a very high accuracy, newer works like [15] or [16] that using state of the art approaches, like Deep Learning methods, achieve over $90 \%$ accuracy in detecting physical activities, using just small wearable devices (like wristbands) with accelerometers and gyroscopes. The issue with these projects is that the methods of training the system are rigid and tightly coupled to the person(s) that performed the activities, being unable to tolerate minor faults.

EmIR tries to overcome the presented issues of current projects and push the state of the art forward. In the next section we present an insight of the robot and cognitive assistant structure and operation.

\section{$3 \quad$ Recommending and following activities}

This section describes the main work developed for this paper. First of all, we detail the different activities that can be recommended to the users. Next, the new version of our low-cost robot, EmIR 3.0, is presented as the interlocutor for the user that is going to recommend the activities. After that, we show how the system makes the following of the activities checking if they have been properly done. First, the ad-hoc hardware to make it so is described and then the artificial neural network to identify the activities is detailed.

\subsection{Activities' Description}

These activities have been selected by physiotherapists, who have helped determine which exercises involve the chest as the main movement. The physiotherapist has recommended five exercises:

1. Wall Push Up. This is a strength-training exercise. Stand at arm's length in front of a wall. Lean forward a little and place the palms of your hands on the wall at shoulder width. Keep your feet still as you slowly move your body toward the wall. Gently push back so your arms are straight (Figure $1)$.

2. Sit to stand. This exercise is good for leg strength. Sit on the edge of the chair, feet apart at the hip. Lean forward slightly. Stand up slowly, using your legs, not your arms. Keep looking forward, not down. Stand up before sitting slowly, from the bottom down (Figure 2).

3. Mini squats Rest your hands on the back of the chair for stability and stand with your feet spread across your hips. Slowly bend your knees as far as is comfortable, keeping them facing forward. Aim to put them on your big toe. Keep your back straight at all times. Gently stand up, squeezing your buttocks as you do so (Figure 3). 


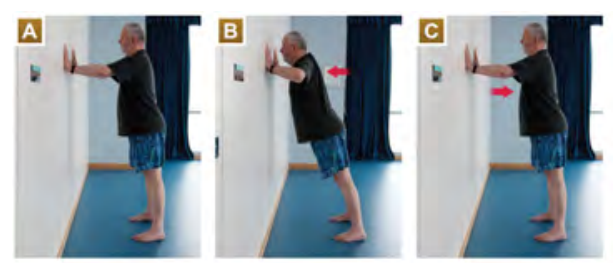

Fig. 1: Wall Push Up Exercise.
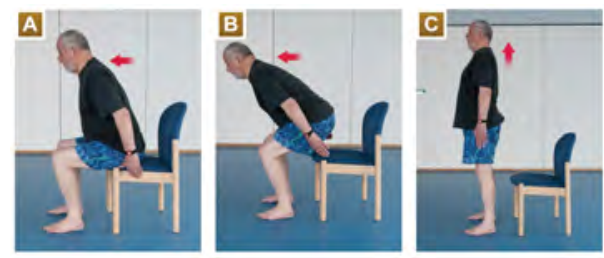

Fig. 2: Sit to Stand Exercise.

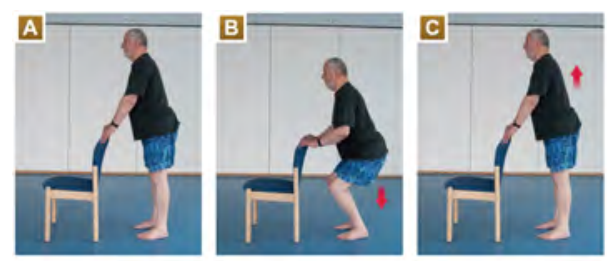

Fig. 3: Mini Squats Exercise. 
4. Back Leg Raises. Slowly lift your right leg backwards, without bending the knees. The chest moves slightly toward the front. Hold this position for one second and then gently lower the leg (Figure 4).

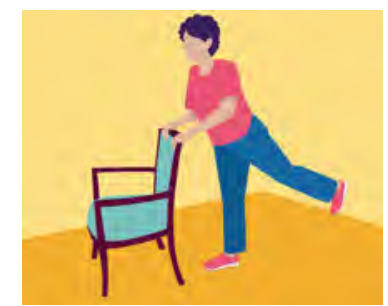

Fig. 4: Back Leg Raises. Copyright (c) 004-2019 Koninklijke Philips N.V. All rights reserved.

5. Clock Reach. Just imagine that you are standing in the center of a clock. The number 12 is directly in front of you and the number 6 is at your back. Hold a chair with your left hand and raise your right leg and extend your right arm so that it points to the number 12 . The return process is done by placing the arm back on the number three and then on the number 12. This process is done with the two arms (Figure 5).

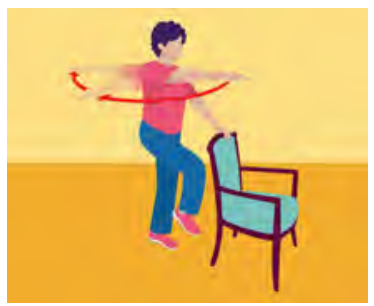

Fig. 5: Clock Reach Exercise. Copyright (c) 004-2019 Koninklijke Philips N.V. All rights reserved.

\subsection{Activities Recommending: EmIR 3.0 -Emotional Intelligent Robot}

This section describes our proposal for a personal trainer robot (Figure 6), capable of recommending, detecting and classifying the physical activities carried out by patients. With the increase of elderly people living alone, and, in some cases, incurring in sedentarism, we see the need to develop systems that recommend 
any kind of physical activity, as it is directly related to welfare, healthy lifestyles, as well as the improvement of health and quality of life.

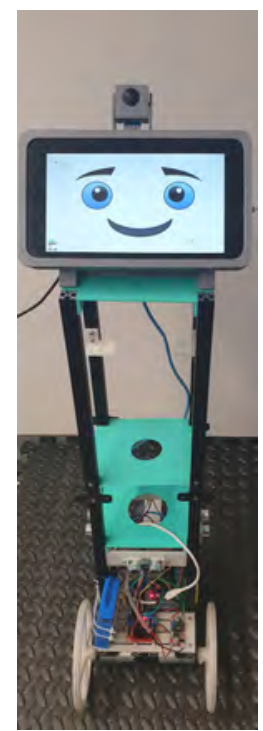

Fig. 6: Prototype of the personal trainer robot.

EmIR is a low cost robot divided into two parts. The first is a low level stage using an Arduino Mega $2560^{3}$, which allows motor control and access to ultrasonic sensors for obstacle detection, allowing the robot to have a reactive behaviour. The second stage is performed using a raspberry pi $3 \mathrm{~b}+$. This allows us to have a higher behaviour as a 7-inch LCD screen, on which it is possible to visualise not only the face of the robot, but also the different physical activities that the robot can recommend. The robot has been built in a modular way, facilitating the incorporation of new elements such as lidars, environmental sensors, etc... The robot also has a camera that allows you to identify people and their emotions [17].

\subsection{Following Activities}

Hardware developed In recent years, different types of internet of things (IoT) devices have been appearing. One of these devices is the one presented by AVNET $^{4}$ that we have used to detect if the activities are properly done.

${ }^{3}$ https://store.arduino.cc/mega-2560-r3

${ }^{4}$ https://www.avnet.com/wps/portal/us/solutions/iot/building-blocks/smartedgeagile/ 
These devices incorporate a wide range of sensors which can be used to acquire various types of data such as accelerometers, or gyroscopes, among others. Using the measurements of acceleration and rotation, it is possible to calculate the linear and angular velocities. These measurements, together with the raw acceleration and gyro measurements, allow the identification of the activities.

As some of the activities to be performed involve chest movement, the sensor was placed in a harness which is used by the patient as can be seen in Figure 7 .

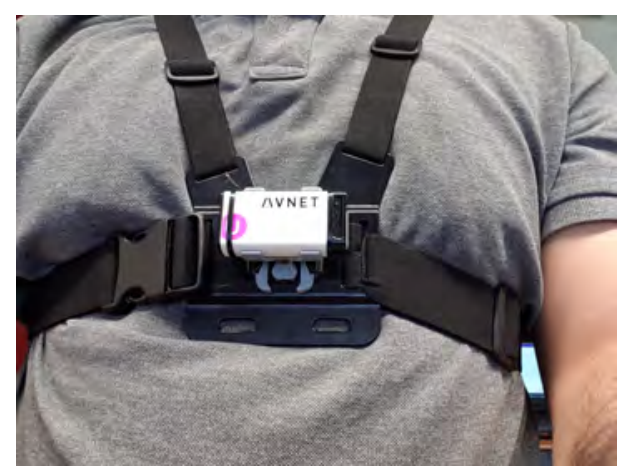

Fig. 7: Sensor and Harness.

Activities Classification Once defined the exercises to classify, the following is to create our dataset. This was necessary because there is no public dataset, which measures these exercises. Ten patients were used to which a total of six repetitions per exercise were captured during 10 sessions, creating a database of 25.000 data. These data were then partitioned into three sub-datasets, one for training with $80 \%$ of the data, $10 \%$ for teas and, finally, $10 \%$ for validation.

Once the dataset was created, it was normalised between $[-1,1]$ so that the dataset is on a common scale. This allows us to avoid distortions, since the characteristics of each measure have different ranges. Once the data was normalised, the next thing to do was to train our network. To do this, the data from our dataset were restructured. Converting them into matrices of 10 columns by 50 rows, where the 10 columns represent the data acquired by the sensor \{Acceleration $(\mathrm{X}, \mathrm{Y}, \mathrm{Z})$ Rotation $(\mathrm{X}, \mathrm{Y}, \mathrm{Z})$ and Linear Speed $(\mathrm{W}, \mathrm{X}, \mathrm{Y}, \mathrm{Z})\}$, while the 50 rows represent the captured samples. This last value can be modified, changing the number of samples to train. In the various experiments that were performed, using 50 rows was the configuration that delivered the best result. This result of our classification of activities can be seen in the confusion matrix (Figure 8). The matrix columns represent the number of predictions for each class, while each row represents the instances in the real class. 
It can be observed that we have a success rate per exercise between 50 to $60 \%$. this is due to small movements generated by patients, movements such as moving to the sides during the performance of exercises. As well as small spasms or involuntary movements. To try to solve this problem, we are getting more samples and we will try to filter or eliminate those involuntary or voluntary movements of patients.

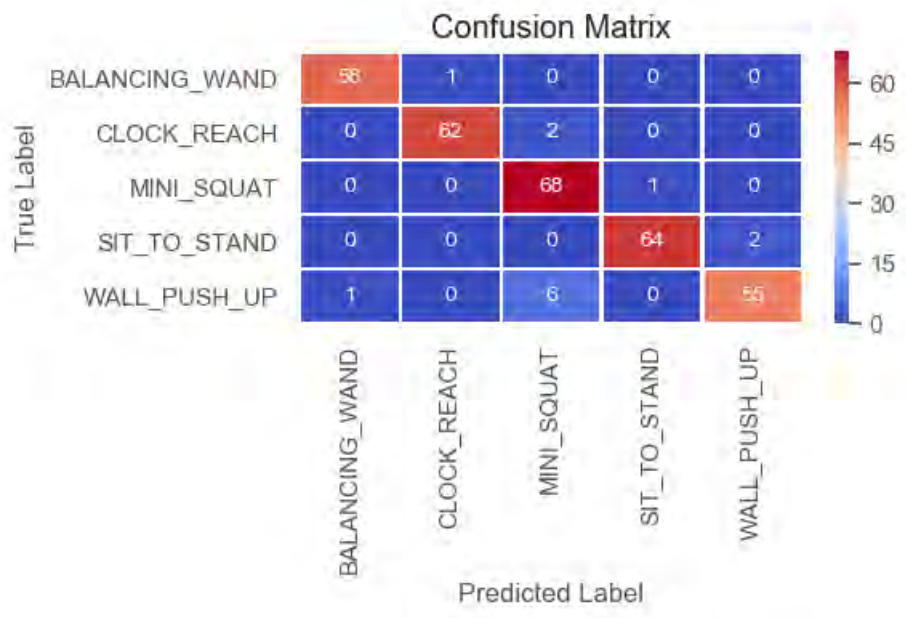

Fig. 8: Confusion matrix obtained from classification.

\section{Conclusions and future work}

In the last decade, the use of robots in home environments has reached a very important role in our society, which no longer perceives the robot as a tool used only in the industrial environment. In this way, robots are acquiring an important role in different scenarios and one of the most important is the care of the elderly. In this paper we have presented a prototype of a robot applied to the care of the elderly. Specifically, the robot is responsible for monitoring the performance of physical exercises, also using a small sensor connected to the robot. In addition, the robot has been added new capabilities for recognition of emotions and recommendation of activities taking into account the result of monitoring the exercises.

As future works, the assistant will be tested by patients and workers of a daycare centre. Specifically, the Centro Social Irmandade de São Torcato in the north of Portugal. The validation will be performed through simple exercises with the patients under the supervision of caregivers. The goal is to improve the physical activities and tasks performed at the centre. 


\section{Acknowledgements}

This work was partly supported by the Spanish Government (RTI2018-095390B-C31) and FCT_-Fundação para a Ciência e Tecnología through the Post-Doc scholarship SFRH/BPD/102696/2014 (A. Costa) and UID/CEC/00319/2019.

\section{References}

1. United Nations, Department of Economic and Social Affairs, Population Division. World population ageing 2015. (ST/ESA/SER.A/390), 2015.

2. Margie E Lachman, Lewis Lipsitz, James Lubben, Carmen Castaneda-Sceppa, and Alan M Jette. When adults don't exercise: Behavioral strategies to increase physical activity in sedentary middle-aged and older adults. Innovation in aging, 2(1):igy007, 2018.

3. Angelo Costa, Vicente Julian, and Paulo Novais, editors. Personal Assistants: Emerging Computational Technologies. Springer International Publishing, 2018.

4. Demetrio Naccari Carlizzi, Giuseppe Quattrone, Chiara Sancin, and Maurizio Turchi. Telemedicine and impact of changing paradigm in healthcare. New Metropolitan Perspectives: Local Knowledge and Innovation Dynamics Towards Territory Attractiveness Through the Implementation of Horizon/E2020/Agenda2030-, 1:39, 2018.

5. J.K. Aggarwal and M.S. Ryoo. Human activity analysis: A review. ACM Comput. Surv., 43(3):16:1-16:43, April 2011.

6. Oscar D Lara and Miguel A Labrador. A survey on human activity recognition using wearable sensors. IEEE communications surveys \& tutorials, 15(3):11921209, 2012.

7. Ester Martinez-Martin and Angel P. del Pobil. Personal Robot Assistants for Elderly Care: An Overview, pages 77-91. Springer International Publishing, Cham, 2018.

8. Martina Čaić, João Avelino, Dominik Mahr, Gaby Odekerken-Schröder, and Alexandre Bernardino. Robotic versus human coaches for active aging: An automated social presence perspective. International Journal of Social Robotics, July 2019.

9. Andre Mateus Joao Garcia Pedro Miraldo Pedro Santos Pedro U. Lima Rodrigo Ventura, Meysam Basiri. A domestic assistive robot developed through robot competitions. In IJCAI 2016 Workshop on Autonomous Mobile Service Robots, 2016.

10. David Portugal, Luis Santos, Paulo Alvito, Jorge Dias, George Samaras, and Eleni Christodoulou. SocialRobot: An interactive mobile robot for elderly home care. In 2015 IEEE/SICE International Symposium on System Integration (SII). IEEE, December 2015.

11. Ester Martinez-Martin and Miguel Cazorla. A socially assistive robot for elderly exercise promotion. IEEE Access, pages 1-1, 2019.

12. Ester Martinez-Martin and Miguel Cazorla. Rehabilitation technology: Assistance from hospital to home. Computational Intelligence and Neuroscience, 2019:1-8, 2019 . 
13. Edmanuel Cruz, Félix Escalona, Zuria Bauer, Miguel Cazorla, José GarcíaRodríguez, Ester Martinez-Martin, José Carlos Rangel, and Francisco GomezDonoso. Geoffrey: An automated schedule system on a social robot for the intellectually challenged. Computational Intelligence and Neuroscience, 2018:1-17, 2018.

14. Jennifer R. Kwapisz, Gary M. Weiss, and Samuel A. Moore. Activity recognition using cell phone accelerometers. ACM SIGKDD Explorations Newsletter, 12(2):74, March 2011.

15. Praneeth Vepakomma, Debraj De, Sajal K. Das, and Shekhar Bhansali. Awristocracy: Deep learning on wrist-worn sensing for recognition of user complex activities. In 2015 IEEE 12th International Conference on Wearable and Implantable Body Sensor Networks (BSN). IEEE, June 2015.

16. Liang Cao, Yufeng Wang, Bo Zhang, Qun Jin, and Athanasios V. Vasilakos. GCHAR: An efficient group-based context - aware human activity recognition on smartphone. Journal of Parallel and Distributed Computing, 118:67-80, August 2018.

17. Jaime Andres Rincon, Alberto Martin, Ângelo Costa, Paulo Novais, Vicente Julián, and Carlos Carrascosa. Emir: An emotional intelligent robot assistant. In AfCAI, 2018. 